ARTICLE

\title{
Machine learning analyses of antibody somatic mutations predict immunoglobulin light chain toxicity
}

Maura Garofalo1, Luca Piccoli (1) 1, Margherita Romeo², Maria Monica Barzago², Sara Ravasio1,3, Mathilde Foglierini (10 ${ }^{1,4}$, Milos Matkovic ${ }^{1}$, Jacopo Sgrignani ${ }^{1}$, Raoul De Gasparo ${ }^{1}$, Marco Prunotto (i) ${ }^{5}$,

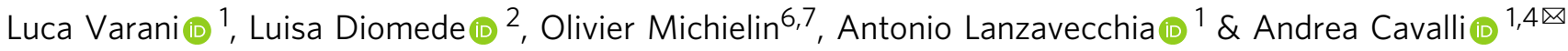

In systemic light chain amyloidosis $(A L)$, pathogenic monoclonal immunoglobulin light chains (LC) form toxic aggregates and amyloid fibrils in target organs. Prompt diagnosis is crucial to avoid permanent organ damage, but delayed diagnosis is common because symptoms usually appear only after strong organ involvement. Here we present LICTOR, a machine learning approach predicting $\mathrm{LC}$ toxicity in $\mathrm{AL}$, based on the distribution of somatic mutations acquired during clonal selection. LICTOR achieves a specificity and a sensitivity of 0.82 and 0.76 , respectively, with an area under the receiver operating characteristic curve (AUC) of 0.87. Tested on an independent set of $12 \mathrm{LCs}$ sequences with known clinical phenotypes, LICTOR achieves a prediction accuracy of $83 \%$. Furthermore, we are able to abolish the toxic phenotype of an LC by in silico reverting two germline-specific somatic mutations identified by LICTOR, and by experimentally assessing the loss of in vivo toxicity in a Caenorhabditis elegans model. Therefore, LICTOR represents a promising strategy for AL diagnosis and reducing high mortality rates in $\mathrm{AL}$.

\footnotetext{
${ }^{1}$ Institute for Research in Biomedicine, Università della Svizzera italiana, Bellinzona, Switzerland. ${ }^{2}$ Department of Molecular Biochemistry and Pharmacology, Istituto di Ricerche Farmacologiche Mario Negri IRCCS, Milan, Italy. ${ }^{3}$ Institute of Microbiology, ETH Zurich, Zurich, Switzerland. ${ }^{4}$ Swiss Institute of Bioinformatics, Lausanne, Switzerland. ${ }^{5}$ School of Pharmaceutical Sciences, Institute of Pharmaceutical Sciences of Western Switzerland, University of Geneva, Geneva, Switzerland. ${ }^{6}$ Molecular Modeling Group, SIB Swiss Institute of Bioinformatics, University of Lausanne, Quartier UNIL-Sorge, Bâtiment Amphipôle, Lausanne, Switzerland. ${ }^{7}$ Department of Oncology, University Hospital of Lausanne, Ludwig Cancer Research - Lausanne Branch, Lausanne, Switzerland. ${ }^{凶}$ email: andrea.cavalli@irb.usi.ch
} 
S ystemic light-chain amyloidosis (AL) is a monoclonal gammopathy characterized by the abnormal proliferation of a plasma cell clone producing large amounts of pathogenic immunoglobulin free light chains $(\mathrm{LCs})^{1}$. LCs, mainly secreted as homodimers ${ }^{2}$, misfold forming toxic species and amyloid fibrils which accumulate in target organs and lead to fatal organ dysfunction and death ${ }^{1}$. Although LCs deposition can occur in any organ except the brain, the kidney and heart are the most affected sites, with the latter bearing the worst prognosis. Symptoms of AL are non-specific and usually reflect advanced organ involvement. Therefore, an early diagnosis is essential to avoid irreversible organ damage. However, the complexity of the disease and its vague symptoms make a timely diagnosis of $\mathrm{AL}$ extremely challenging 3,4 .

Pre-existing monoclonal gammopathy of undetermined significance (MGUS) is a known risk factor for developing AL, with $9 \%$ of MGUS patients progressing to $\mathrm{AL}^{5-7}$. However, early diagnosis is still difficult since reliable diagnostic tests predicting whether MGUS patients are likely to develop AL are currently lacking ${ }^{7,8}$. Predicting the onset of $\mathrm{AL}$ is highly challenging, as each patient carries a different pathogenic LC sequence resulting

$1 a$

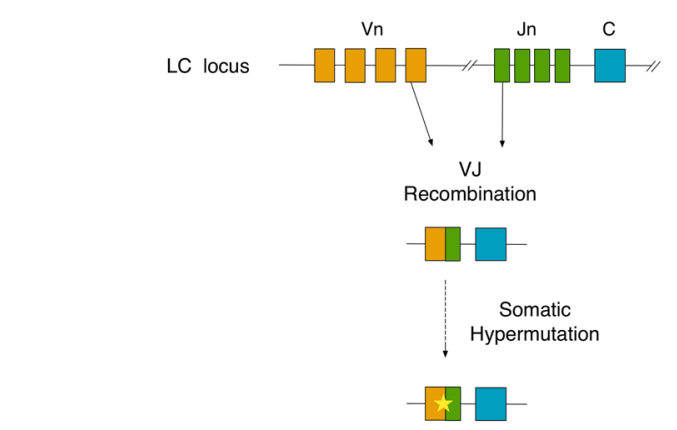

1b

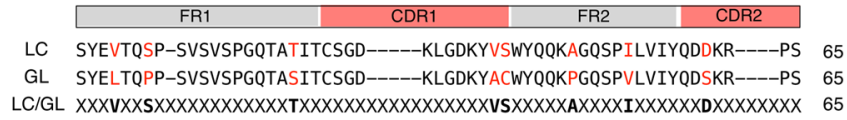

from a unique rearrangement of variable $(\mathrm{V})$ and joining $(\mathrm{J})$ immunoglobulin genes and a unique set of somatic mutations (SMs) acquired during $B$ cell affinity maturation ${ }^{9}$ (Fig. 1a). Therefore, the development of a specific prediction tool represents a crucial step to anticipate $\mathrm{AL}$ diagnosis and improve patients' prognosis.

Machine learning techniques are becoming very prominent in different areas of science and are also gaining acceptance in medicine. Indeed, machine learning has been used in different areas of medicine, such as diagnosis ${ }^{10-12}$, prognosis ${ }^{13,14}$, drug discovery ${ }^{15,16}$ and drug sensitivity prediction ${ }^{17-19}$. In these approaches, machines learn information from data without being explicitly programmed and simulate human intelligence to make predictions ${ }^{20}$. The high diversity of LC sequences accountable for AL development and the possibility of accessing databases of pathogenic and non-pathogenic LC sequences prompted us to use a machine-learning-based strategy to devise a predictor of LC toxicity in AL named LICTOR ( $\lambda$-LIght-Chain TOxicity predictoR).

LICTOR uses SMs as predictor variables based on the hypothesis that SMs are the main LC toxicity-discriminating
16

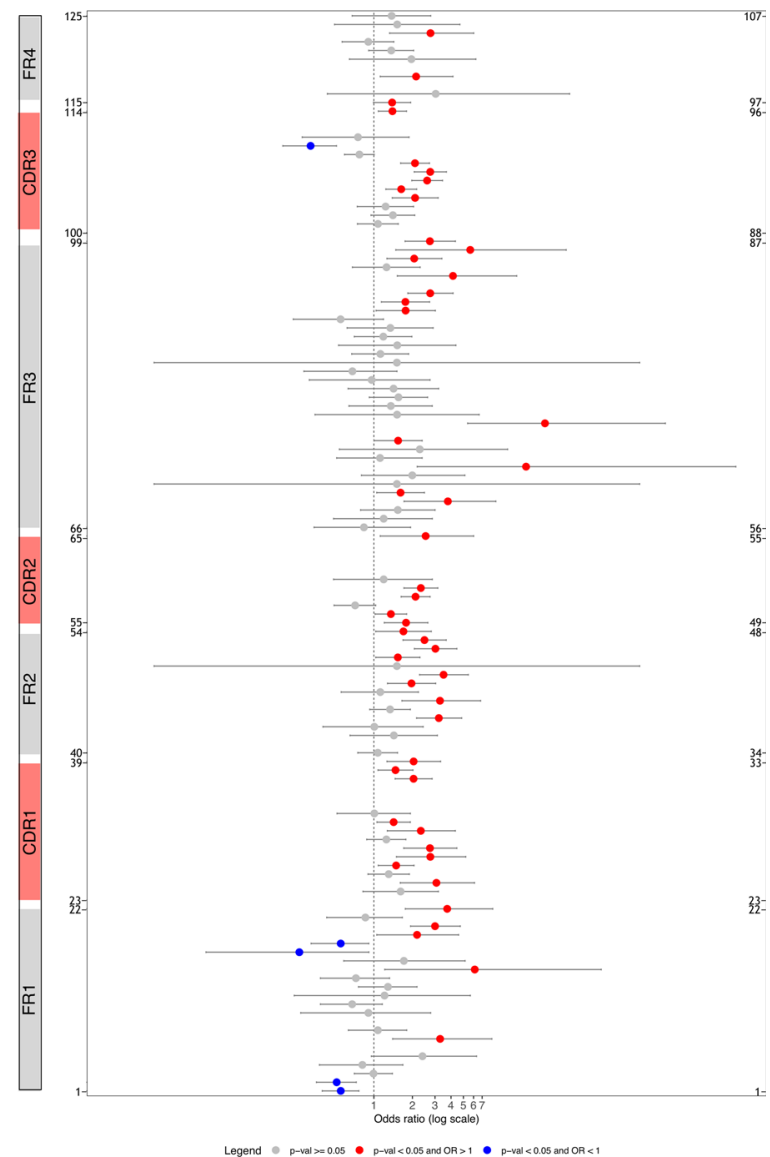

Fig. 1 The presence of SMs differentiates toxic and non-toxic LC sequences. a Schematic representation of the generation of LC diversity through the processes of VJ recombination and somatic hypermutation. b Alignment of an LC sequence with the corresponding germline (GL) sequence according to Kabat-Chothia scheme using a progressive enumeration for a total of 125 positions ("Methods"). Structural elements of immunoglobulin light chains are depicted on top of the sequences (FR1 = framework 1, CDR1 = complementary determining region 1, FR2 = framework 2, CDR2= complementary determining region 2, FR3 = framework 3, CDR3 = complementary determining region 3, FR4 = framework 4). Residues in red depict somatic mutations (SMs). The third line shows the encoding scheme used by the classifier with SMs (displayed in bold) and unmutated positions represented by an " $X$ ". c Data are presented as odds ratio (OR) and their 0.95 confidence interval (grey horizontal bars) for all 125 positions of the LC sequences according to our sequential numbering scheme ( $y$-axis). The corresponding Kabat-Chothia enumeration is reported on the right. Structural elements of immunoglobulin light chains are shown on the left. ORs for positions with no statistically significant difference between tox and nox sequences $(p \geq 0.05)$ are represented as grey dots. Positions with statistically significant differences $(p<0.05)$ are depicted as either red $(O R>1)$ or blue $(O R<1)$ dots. Fisher's exact test was used as statistical test. . 
factors. We assess LICTOR's performance with an independent set of LCs with a known clinical phenotype but not used in the training. Furthermore, to experimentally validate LICTOR, we use our predictor to abolish the pathological phenotype of a cardiotoxic LC and verify the outcome with a Caenorhabditis elegans-based assay evaluating the reduction of the pharyngeal pumping rate after administration of cardiotoxic LCs as a measure of proteotoxicity. Taken together, these results confirm that LICTOR provides insights into specific features differentiating toxic and non-toxic LCs. Therefore, it may represent a powerful tool to improve AL diagnosis and unveil a novel strategy for patient treatment through personalized medicine.

\section{Results}

SMs are key LC toxicity-discriminating factors. To investigate the role of SMs in the generation of toxic LCs and validate their use as predictor variables in an LC toxicity predictor, we collected a database of $1075 \lambda$ LC sequences. The database included 428 "toxic" sequences (i.e. LCs responsible for the formation of toxic aggregates an $\mathrm{AL}$ development) extracted from $\mathrm{AL}$ patients (hereafter referred to as tox) and 647 "non-toxic" LCs (nox) comprising sequences from healthy donor repertoires, other autoimmune diseases or cancer, obtained from Amyloid Lightchain Database (AL-Base) ${ }^{21}$ (428 tox, 590 nox) and an in-house LCs' database not related to AL (57 nox). We restricted our analysis to $\lambda$ LCs since this isotype is more prevalent than the kappa ( $\kappa)$ isotype in AL patients $(\lambda / \kappa=3: 1$ compared to that of healthy individuals, $\lambda / \kappa=1: 2)^{22}$. To identify SMs, all LCs were aligned to the corresponding germline (GL) sequence obtained using the IMGT database $\mathrm{e}^{23}$. LCs were then numbered according to the Kabat-Chothia scheme (using a progressive enumeration from 1 to 125), allowing the structural comparison of LCs with different sequence lengths ("Methods" and Fig. 1b). Next, we counted the number of mutated $(\mathrm{M})$ and non-mutated $(\mathrm{NM})$ residues at each position $i$ in tox and nox sequences ( $\operatorname{tox}^{i}{ }_{\mathrm{M}}$ and $\operatorname{nox}^{i}{ }_{\mathrm{M}}$, tox ${ }_{\mathrm{NM}}{ }_{\mathrm{NM}}$ and nox $^{i}{ }_{\mathrm{NM}}$, respectively) and used Fisher's exact tes $\mathrm{t}^{24}$ to assess whether the frequencies of mutations $\left(\operatorname{tox}_{M}^{i}, \operatorname{nox}_{M}^{i}\right)$ and nonmutations (tox ${ }_{\mathrm{NM}}^{i}$, nox $\left.^{i}{ }_{\mathrm{NM}}\right)$ were significantly $(p<0.05)$ different. Finally, the odds ratio $(\mathrm{OR})^{24}$ was used to assess the association strength between mutations and toxicity at each position $i$ in tox and nox sequences ("Methods" and Fig. 1c). Interestingly, 48 of 53 positions with a statistically significant difference $(p<0.05)$ between the two groups (Fig. 1c) showed a higher rate of mutation in the to $x$ group $(\mathrm{OR}>1)$, while only 5 positions reported a higher mutation rate in the nox group $(\mathrm{OR}<1)$. To exclude possible bias induced by the use of a group of nox sequences having an artificially low level of SMs, we randomly selected 1000 LC sequences from a healthy donor repertoire (hdnox) $)^{25}$ and compared the probability distributions of the number of SMs (PDSM) between the three groups. We observed similar PDSM between the nox and $h d n o x$ groups, while the PDSM of tox and hdnox, as well as, tox and nox were significantly different. This result supports nox sequences as a bona fide group of LCs (Supplementary Fig. 1). Overall, these findings suggest that SMs are key determinants of the toxicity of LCs and, thus, can be used as predictor variables to develop LC toxicity prediction tools.

Prediction of LC toxicity using machine learning. The previous findings prompted us to use SMs as features to develop a machine learning approach automatically classifying LCs as either toxic or non-toxic in AL. To this end, we combined the information from SMs with knowledge of the 3D structure of LC homodimers ${ }^{26,27}$ to create three families of predictor variables used in the training of machine learning algorithms. The first family, termed AMP (amino acid at each mutated position), highlights sequence features, identifying the presence or absence of an SM at each position of the LC sequences. The second family, termed MAP (monomeric amino acid pairs), identifies the presence or absence of mutations in residues in close contact in the LC monomeric 3D structure (distance $<7.5 \AA$ ). Finally, the third family, named DAP (dimeric amino acid pairs), identifies the presence or absence of mutations at positions in close contact, but belonging to different chains. Next, four machine learning algorithms (Bayesian network, logistic regression, J48 and random forest $)^{28}$ were evaluated for their ability to solve the classification problem, using our database as input. To assess the importance of the different classes of predictor variables, we performed 28 prediction experiments including all the possible combinations of AMP, MAP and DAP families. In addition, to avoid unbalanced class problems, i.e. the tendency of machine learning algorithms to assign sequences to the largest class in the dataset, nox in our case, each of the 28 experiments was performed with and without balancing the training set using an SMOTE (synthetic minority over-sampling technique) filter ${ }^{29}$. The assessment of algorithms and predictor variable combinations was performed using 10 -fold cross-validation to avoid overfitting. We found that for all the tested machine learning algorithms, the best combination of predictor variable families provided an area under the receiver operating characteristic curve (AUC) that substantially differed from that of a random classifier (0.50), with random forest being the best classifier (0.87) and J48 being the worst (0.75) (Fig. 2a and Supplementary Data 1). Furthermore, all four classifiers relied on the AMP family to predict LC toxicity, while only random forest used all three families of predictor variables $(\mathrm{AMP}+\mathrm{MAP}+\mathrm{DAP})$ in its best configuration. Overall, these findings highlight the importance of the structural context of SMs in defining the toxicity of an LC and identify random forest using AMP, MAP and DAP as the best approach in our case. For this reason, we used random forest in our implementation of LICTOR.

Validation of LICTOR. Next, we sought to validate the prediction accuracy of LICTOR with a set of sequences with known clinical phenotypes but not present in the training set (valset) ${ }^{27}$. The valset (Supplementary Data 2) comprised a total of 12 LCs, including 7 sequences associated with AL with cardiac involvement $(\mathrm{H} 3, \mathrm{H} 6, \mathrm{H} 7, \mathrm{H} 9, \mathrm{H} 15, \mathrm{H} 16$ and $\mathrm{H} 18)$ and 5 from multiple myeloma (MM) patients (M2, M7, M8, M9 and M10). LICTOR was able to correctly classify 10 (6 tox and 4 nox) of the 2 LCs as either toxic or non-toxic (Supplementary Data 2 and Fig. 3a). The probability of achieving a similar accuracy with a random classifier was 0.016 , strengthening the argument that LICTOR is a robust and accurate tool to predict the clinical toxicity of previously unseen LCs.

To further assess the robustness of LICTOR, we performed two additional tests. In the first test, we predicted the toxicity of 100 randomly selected LCs from the healthy donor repertoire $h d n o x$ (all absent from the training set). In this case, LICTOR correctly classified $80 \%$ of the sequences as non-toxic (Supplementary Data 3), thus confirming a similar level of accuracy as for the training set. In the second test, we assessed LICTOR by further verifying the absence of overfitting. To achieve this, we took all tox sequences and randomly labelled half of them as nox. Then, we trained a classifier using the 10 -fold cross-validation on such a dataset obtaining an AUC of 0.5 (Supplementary Data 4), equivalent to that of a random classifier. The same procedure has been used for the nox group (by randomly assigning half of them as tox and training a classifier on such a dataset), obtaining, in this case also, an AUC of 0.5 (Supplementary Data 5). These 
2a

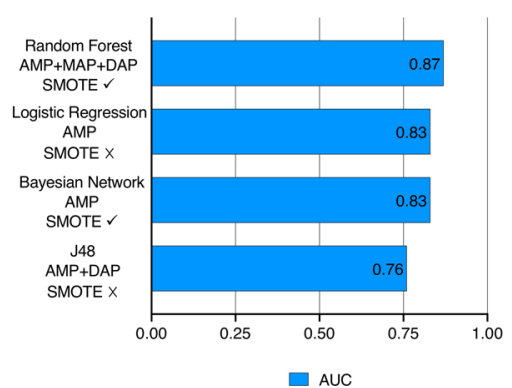

$2 b$

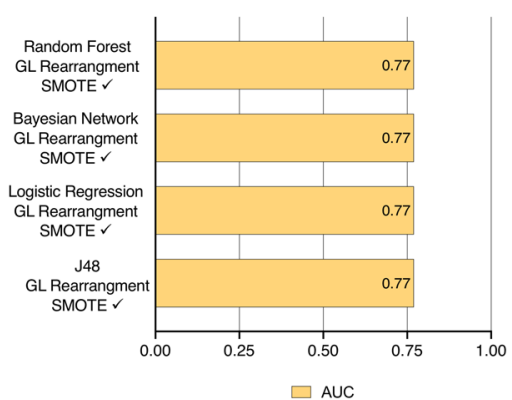

$2 c$

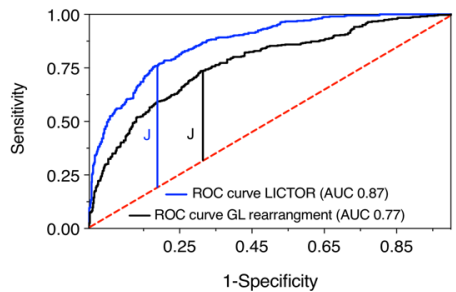

2d

\begin{tabular}{|c|c|c|c|}
\hline Ranking & AMP & MAP & DAP \\
\hline 1 & $\begin{array}{c}49 \mathrm{~A} \\
54 / 8^{\mathrm{a}}\left|2.8 \mathrm{e}-15^{\mathrm{b}}\right| 1^{\mathrm{c}}\end{array}$ & $\begin{array}{c}104 X-108 X \\
178 / 410|2.9 e-12| 9\end{array}$ & $\begin{array}{c}49 \mathrm{~A}-116 \mathrm{X} \\
53 / 8|6.6 \mathrm{e}-15| 2\end{array}$ \\
\hline 2 & $\begin{array}{c}107 x \\
265 / 529 \mid 8.3 e-13 \text { | } 8\end{array}$ & $\begin{array}{c}44 X-98 X \\
336 / 599|6.2 e-11| 13\end{array}$ & $\begin{array}{c}65 X-107 X \\
250 / 520|9.8 e-15| 3\end{array}$ \\
\hline 3 & $\begin{array}{c}106 \mathrm{X} \\
247 / 505|1.8 \mathrm{e}-12| 11\end{array}$ & $\begin{array}{c}52 X-65 X \\
323 / 585|6.2 e-11| 13\end{array}$ & $\begin{array}{c}4 X-49 A \\
53 / 8|6.6 e-15| 4\end{array}$ \\
\hline 4 & $\begin{array}{c}108 \mathrm{H} \\
34 / 5|6.4 \mathrm{e}-10| 19\end{array}$ & $\begin{array}{c}44 X-99 X \\
310 / 571|8.9 e-11| 14\end{array}$ & $\begin{array}{c}49 X-99 X \\
296 / 573|5.7 e-15| 5\end{array}$ \\
\hline 5 & $\begin{array}{c}78 x \\
401 / 645|1.5 e-09| 22\end{array}$ & $\begin{array}{c}54 X-58 X \\
193 / 419|2.6 e-10| 16\end{array}$ & $\begin{array}{c}52 X-108 X \\
161 / 400|7.6 e-15| 6\end{array}$ \\
\hline 6 & $\begin{array}{c}106 \mathrm{~N} \\
65 / 28|1.4 \mathrm{e}-09| 24\end{array}$ & $\begin{array}{c}1 \mathrm{X}-108 \mathrm{H} \\
28 / 2|6.3 \mathrm{e}-10| 17\end{array}$ & $\begin{array}{c}49 \mathrm{~A}-99 \mathrm{X} \\
51 / 8|6.9 \mathrm{e}-14| 7\end{array}$ \\
\hline 7 & \begin{tabular}{c|c|c} 
& $49 \mathrm{X}$ \\
$353 / 610$ & $1.3 \mathrm{e}-09$ | 28
\end{tabular} & $\begin{array}{c}44 \mathrm{H}-96 \mathrm{X} \\
61 / 24|6.1 \mathrm{e}-10| 20\end{array}$ & $\begin{array}{c}3 \mathrm{X}-49 \mathrm{~A} \\
43 / 6|1.5 \mathrm{e}-12| 10\end{array}$ \\
\hline 8 & $\begin{array}{c}44 X \\
345 / 602|1.3 e-09| 29\end{array}$ & $\begin{array}{c}44 X-97 X \\
317 / 575|5.2 e-10| 21\end{array}$ & $\begin{array}{c}66 X-107 X \\
263 / 516|7.0 e-11| 15\end{array}$ \\
\hline 9 & $\begin{array}{c}52 \mathrm{X} \\
337 / 594 \mid 1.5 \mathrm{e}-09 \text { | } 31\end{array}$ & $\begin{array}{c}42 X-52 X \\
323 / 580|1.0 e-09| 25\end{array}$ & $\begin{array}{c}49 X-116 X \\
350 / 609|3.5 e-10| 18\end{array}$ \\
\hline 10 & 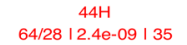 & $\begin{array}{c}56 X-59 X \\
211 / 493\left|2.2 e^{-16}\right| 23\end{array}$ & $\begin{array}{c}52 \mathrm{X}-116 \mathrm{X} \\
334 / 592|8.2 \mathrm{e}-10| 23\end{array}$ \\
\hline
\end{tabular}

2e
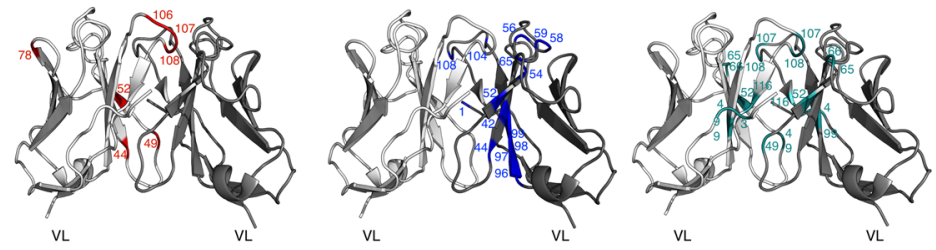

Fig. 2 Machine learning predicts toxic and non-toxic sequences and identifies key features of toxicity. a AUC of the best configuration for each of the considered machine learners (blue bars). Different combinations of three families of predictor variables were tested, with $(\boldsymbol{V})$ or without $(\boldsymbol{X})$ the SMOTE balancing technique. $\mathbf{b}$ The yellow bars show the best AUC value obtained by each machine learner using only the LC germline VJ rearrangements as predictor variables. c ROC curve for LICTOR (i.e. random forest using AMP + MAP + DAP) compared with a predictor (random forest) using only the LC germline VJ rearrangements as predictor variables. $\mathbf{d}$ Top 10 features of each family ranked by information gain. Each feature is enumerated according to our sequential numbering scheme, while the corresponding Kabat-Chothia enumeration for each feature is reported in parenthesis. Kabat-Chothia insertions are reported with lowercase letters. Below each predictor variable are shown the occurrence in tox/nox sequences (a), the $p$-value (b) and the feature selection general ranking (c) (red = AMP features, blue = MAP features and green = DAP features). e Mapping of the top 10 features of each family on the variable domains of an LC homodimeric structure (PDB ID: 2OLD, represented in white and grey in cartoon). AMP features are shown in red in the left image, MAP features in blue in the middle image and DAP features in green in the right image. The colour code used in the table to represent the three feature families is maintained in their structural representation in (d).

results further underline that tox and nox sequences have distinctive features allowing their discrimination.

LC germline VJ rearrangement information does not improve prediction performance. To further underscore the role of SMs as main discriminants between toxic and non-toxic LCs, we trained the same machine learners employed before, using the LC germline VJ rearrangements as a unique predictor variable, given the well-documented overrepresentation of certain VL germline genes in $\mathrm{AL}^{30-32}$. All the resulting germline-based classifiers achieved an AUC of 0.77 in their best configuration (Fig. $2 \mathrm{~b}$ and Supplementary Data 6), a value substantially better than that of a random classifier, although much lower than LICTOR's score (0.87). Interestingly, adding LC germline VJ rearrangements to LICTOR did not improve its prediction performance (Supplementary Data 7).

Next, we computed the specificity and sensitivity of the two random forest predictors, LICTOR and the germline-based predictor, maximizing the Youden index $(J)^{33}$ as a function of the confidence level of the random forest predictions, i.e. the probability that a sequence belongs to the predicted phenotype (Fig. 2c). LICTOR achieved a specificity of 0.82 and a sensitivity of $0.76(J=0.58$, threshold $=0.46$ in identifying tox $)$, while the germline-based classifier showed a specificity of 0.69 and a sensitivity of $0.73(J=0.43$, threshold $=0.48$ in identifying tox $)$. Overall, these data suggested that SMs harbour key information that can be used to discriminate between tox and nox, while LC germline VJ rearrangements do not seem to carry additional information that can improve the prediction performance of LICTOR.

LICTOR unveils specific features of LC toxicity. To identify the key features leading to LC toxicity in $\mathrm{AL}$, we ranked the predictor variables of LICTOR according to their "information gain", a value representing the importance of the information carried by each predictor variable for the classification ${ }^{34}$. We found that among the top 10 most important features of the three families of predictor variables, feature $49-\mathrm{A}$, which denotes an SM to alanine 
3a

Clinical Phenotype Clinical Phenotype Non-Toxic $\quad$ Cardiotoxic
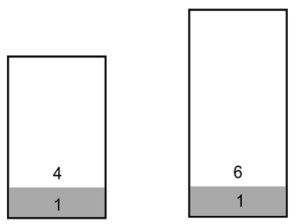

$\square$ LICTOR correct predictions $\square$ LICTOR incorrect predictions 3d

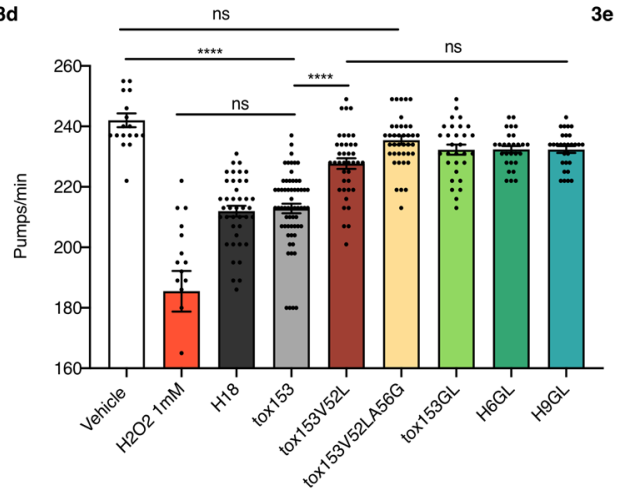

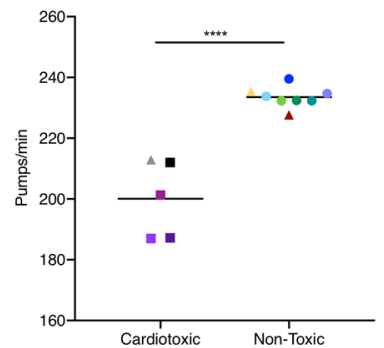

- $\begin{aligned} \mathrm{H} 18 \\ \mathrm{H} 7\end{aligned}$

- $\begin{array}{r}\mathrm{H} 6 \\ \mathrm{H} 9\end{array}$

- M8

- $\mathrm{M} 2$

- HGGL

$\Delta$ tox153

tox153 V52L
tox153 V52LA56G
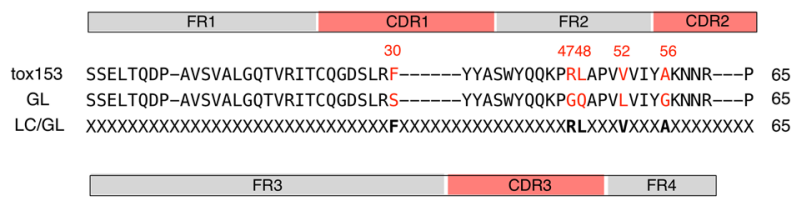

tox153 SGIPDRFSGSS--SGNTASLTITGAQAEDEADYYCNSRDSSGNH----VVFGGGTKLTVL 125 GL SGIPDRFSGSS--SGNTASLTITGAQAEDEADYYCNSRDSSGNH----VVFGGGTKLTVL 125 LC/GL $X X X X X X X X X X X X X X X X X X X X X X X X X X X X X X X X X X X X X X X X X X X X X X X X X X X X X X X X X X X X X X \quad 125$ 3c

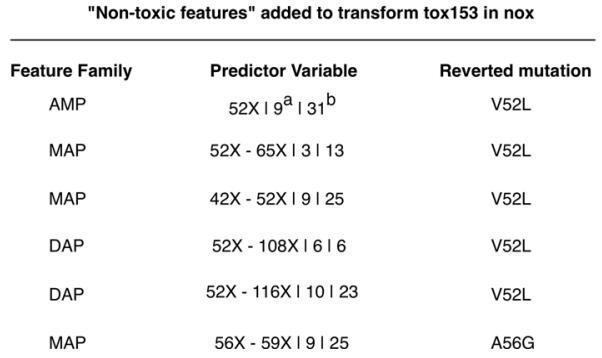

Fig. 3 LICTOR accurately predicts the LC toxicity of sequences absent from the training set and is able to revert the pathological phenotype of a cardiotoxic LC. a LICTOR predictions based on an independent set of LCs, i.e. not present in the training set. Toxic LCs are from patients affected by AL with cardiac involvement, while non-toxic LCs are from patients with multiple myeloma (see also Supplementary Data 2). Predictions are divided according to the clinical phenotype. White part of the bars represent correct LICTOR predictions, while grey part of the bars represent incorrect predictions.

b Sequence of a cardiotoxic LC (tox153) used to neutralize the toxic phenotype using LICTOR and the non-toxic features unveiled by feature selection. Tox153 is aligned with the corresponding germline (GL) sequence, and the third line shows the difference in somatic mutations (SMs) between the LC and the GL sequence ( $L C / G L)$ with SMs (displayed in bold) and unmutated positions represented by an " $X$ ". c The table represents the non-toxic features according to information gain used to revert the toxic phenotype of tox153. For each predictor variable, we also report the ranking in the specific-feature family $\left({ }^{a}\right)$ and the feature selection general ranking $\left({ }^{b}\right)$. d Proteotoxic effect of tox 153 protein, of the two mutants in silico designed by adding non-toxic features (tox153V52L and tox153V52LA56G) and of the tox153 GL protein. The proteotoxic effect of H18 cardiotoxic LC and of the GL proteins H6GL and $\mathrm{H} 9 \mathrm{GL}$ are tested as well. Proteins in $10 \mathrm{mM} \mathrm{PBS}(100 \mu \mathrm{g} / \mathrm{mL})$ were administered to $\mathrm{C}$. elegans $(100 \mu \mathrm{L} / 100$ worms $)$. Vehicle $(10 \mathrm{mM} \mathrm{PBS})$ and $1 \mathrm{mM} \mathrm{H} \mathrm{O}_{2}$ were administered as negative and positive controls, respectively. Pharyngeal activity was determined $24 \mathrm{~h}$ after treatment by determining the number of pharyngeal bulb contractions (pumps/min). Data are the pumps/min in mean \pm SE ( $n=30$ worms/assay, two assays). ${ }^{\star \star \star \star} p<0.0001$ one-way ANOVA, Dunn's post hoc test. e Values of pharyngeal bulb contraction (pumps/min) of some LCs (H6, H7, H9 and M2, M7, M8) listed in Supplementary Data 2 are from AL patients with cardiac involvement (cardiotoxic) and patients with multiple myeloma (non-toxic). These values were previously obtained under the same experimental conditions employed in this study 35,36 . Additionally, values of pharyngeal bulb contraction (pumps/min) of $\mathrm{H} 18, \mathrm{H} 6 \mathrm{GL}$ and $\mathrm{H} 9 \mathrm{GL}$ are reported as well ( $n=30$ worms/assay, two assays). Each square is the mean value for H6, H7, H9 and H18 while dots represent the mean of M2, M7, M8, $\mathrm{H} 6 \mathrm{GL}$ and H9GL. Horizontal lines represent the mean of cardiotoxic and non-toxic LCs ( ${ }^{\star \star \star \star} p<0.0001$, two-tailed unpaired $t$-test). The values of pumps/ min obtained after the administration of tox153, tox153V52L and tox153V52LA56G are also plotted (triangles).

at position 49, obtained the highest score in the AMP family ranking, as well as in the general ranking (Fig. $2 \mathrm{~d}$ and Supplementary Data 8). Indeed, feature 49 -A was present in 54 tox sequences but only 8 nox sequences. Furthermore, the 49-A mutation, which is located at the dimeric interface of LCs (Fig. 2e), was also ranked among the top 10 features in the DAP family in combination with no substitutions at other residue positions (Fig. 2d). Moreover, among the best-ranked predictor variables of the three families, those describing mutated positions were more frequent in tox sequences than in nox sequences (Fig. 2d). Interestingly, all these mutations were located at the LC homodimeric interface (Fig. 2e), suggesting that mutations in these positions may affect the structural integrity of the dimeric interface and/or induce local instability of the monomer, thus leading to LC misfolding and aggregation. A similar trend was also observed for other top-ranked features, where unmutated positions were, conversely, more frequent in nox sequences than in tox sequences (Fig. $2 \mathrm{~d}$ ).

To investigate the role of the top-ranked features in the prediction of LC toxicity, we performed a quantitative analysis of the importance of features identified by the feature selection technique. To this end, we trained 30 different classifiers (with a 10 -fold cross-validation) adding successively the 10 most important features of each feature family according to their information gain. Results are reported in Supplementary Fig. 2 and Supplementary Data 9. Interestingly, the classifier using only the highest ranked feature $49-\mathrm{A}$ achieves an accuracy of $64 \%$ with an AUC of 0.55 , while to achieve an AUC above 0.77 at least 17 top features are required.

Taken together, these findings show that the presence or absence of specific mutations at specific positions of an LC are key features used by LICTOR to classify the LC phenotype. More importantly, this further underlines the pivotal role of SMs as causative of AL.

Reverting the toxic phenotype of an LC using LICTOR. Having assessed the prediction accuracy of LICTOR, we sought to validate the key toxicity determinants identified previously through information gain by computationally reverting the toxic 
phenotype of an LC with LICTOR and verifying the results in a validated in vivo C. elegans model ${ }^{35,36}$. We therefore selected an LC from our database (tox153) previously described in the literature as cardiotoxic ${ }^{37}$, and thus having the worst prognosis in AL. For this sequence, we had access to a bone marrow sample, from which we obtained the full-length sequence of tox153 (see "Methods"). Despite its toxic phenotype, tox 153 differs by only 5 SMs from the corresponding germline (Fig. 3b), hence representing a good candidate with which to perform our study. From the analysis of the 5 SMs of tox 153 , we found that the best candidate, i.e. the mutation with the largest information gain, was at position 52, in which the germline leucine (LEU) was somatically mutated to a valine (VAL). This feature was one of the topranked predictor variables in all three families (AMP, MAP and DAP). In fact, an unmutated amino acid at position 52 (52X) was significantly more frequent in nox sequences than in tox sequences ( $p_{\text {val }}=1.5$ e-09, Fig. $\left.2 \mathrm{~d}\right)$. Therefore, $52 \mathrm{X}$ may represent a "non-toxic feature" and is able to revert the phenotype of tox153 (Fig. 3c). To test this hypothesis, we restored the leucine of the germline sequence at position 52 of tox 153 (tox153V52L) and used LICTOR to predict the toxicity of the new sequence. LICTOR predicted tox153V52L as a nox sequence, highlighting that this single point mutation is able to completely revert the toxic phenotype of tox153, according to in silico prediction. Next, we analysed the other 4 SMs according to their information gain. Among these SMs, only the MAP feature 56X-59X $\left(p_{\text {val }}=2.2 \mathrm{e}-\right.$ 16, Fig. 2d) was included in the top rank. Since tox 153 is mutated at position 56 but not at position 59, we also reverted this SM in tox153V52L by mutating alanine to glycine (tox153V52LA56G) (Fig. 3c). Interestingly, in silico prediction by LICTOR confirmed the non-toxic phenotype of tox153V52LA56G. These results underline that key predictor variables identified in the feature selection process and used by LICTOR to perform the predictions represent molecular determinants of AL.

Experimental validation of LICTOR using C. elegans. Next, we assessed the accuracy of LICTOR's toxicity predictions in a validated in vivo model, exploiting the ability of $C$. elegans to specifically identify cardiotoxic $\mathrm{LCs}^{35,36}$. To this end, recombinantly expressed tox153, single mutant tox153V52L, double mutant tox153V52LA56G and tox153 germline protein (tox153GL), the protein without SMs, were administered to worms and their toxicity was evaluated by measuring alterations in the pharyngeal pumping rate ${ }^{35,36}$. Tox153 caused significant pharyngeal dysfunction (Fig. 3d) comparable to that induced by the administration of cardiotoxic LCs purified from patients suffering from AL (H6, H7, H9) (Fig. 3e) 35,36,38, while the effect of tox153GL was comparable to that of the vehicle. The presence of a single mutation in tox153V52L significantly decreased the ability of the wild-type protein to cause pharyngeal toxicity (Fig. 3d). Notably, the double mutant tox153V52LA56G, similar to LCs purified from patients affected by MM (M2, M7, M8) ${ }^{35,36}$, did not display toxic activity (Fig. 3e).

Finally, we thought to validate our starting hypothesis that LCs acquire toxic features trough the addition of specific SMs during the process of affinity maturation and that, conversely, germline LCs are never associated with AL development. To achieve this, we exploited the $C$. elegans model and tested a total of recombinantly expressed three germline LCs (H6GL, H9GL and Tox153GL). Values of their pharyngeal toxicity were then compared to those of the corresponding cardiotoxic LCs (H6, H9 and tox153), for which the pharyngeal bulb contraction (pumps/min) values were already published ${ }^{35,36}$ and obtained under the same experimental conditions, or measured by us (Fig. 3d, e). Interestingly, the three germline LCs do not show any significant proteotoxicity. Finally, we tested an additional cardiotoxic LC (H18) belonging to the same germline family of tox153 (Fig. 3d), whose sequence was present in the valset. As expected, H18 caused a significant impairment of C. elegans pharyngeal activity, while not all germline proteins affect the pharyngeal pumping rate (Fig. $3 \mathrm{~d}, \mathrm{e}$ ).

Globally, these results experimentally validate our starting hypothesis that SMs are pivotal determinants of LC toxicity. Moreover, our in vivo analysis confirms the soundness of LICTOR predictions and the validity of key predictor variables identified by information gain as determinants of in vivo proteotoxicity.

\section{Discussion}

Early diagnosis of AL is essential to readily apply therapeutic interventions and prevent permanent and fatal organ damage. However, AL is usually detected only once the symptoms reflecting advanced organ involvement occur, which results in poor patient prognosis. Moreover, although pre-existing MGUS is a known risk factor for AL, predicting whether MGUS patients will progress to AL remains an open, unsolved problem. The extreme sequence diversity of LCs responsible for AL, due to VJ recombination and SMs, further complicates this scenario. Consequently, to deepen our understanding of the AL determinants and ultimately foster early $\mathrm{AL}$ diagnosis, we investigated LC sequences with a known clinical phenotype, with the aim of devising a predictive tool that can flag toxic LCs in advance (i.e. LCs responsible for the formation of toxic aggregates and $\mathrm{AL}$ development). To achieve this goal, we analysed a large dataset of toxic (tox) and non-toxic (nox) LCs of the $\lambda$ isotype, the most frequent isotype in $\mathrm{AL}$, following the hypothesis and also posed by other research groups ${ }^{39-41}$ that specific SMs can increase the propensity of LCs to cause AL. Therefore, we performed a statistical analysis of the distribution of SMs between tox and nox sequences. This analysis revealed that toxic LCs have significantly higher SM frequencies than non-toxic LCs (Fig. 1c). Based on these findings, we designed LICTOR, a machine learning approach using SMs to classify the LC phenotype. LICTOR achieved a specificity and a sensitivity of 0.82 and 0.76 , respectively, with an AUC of 0.87, making it an unprecedented tool in early $\mathrm{AL}$ diagnosis. Interestingly, including LC germline $\mathrm{VJ}$ rearrangements as additional predictor variables in LICTOR configuration did not improve prediction performance, further suggesting that, despite the prevalence of some VL germline genes in AL, SMs represent the critical driver of the disease.

LICTOR differs from approaches such as AGGRESCAN ${ }^{42}$, PASTA $^{43}$, WALTZ ${ }^{44}$ and others ${ }^{45-48}$ that predict the aggregation propensity of a protein by identifying amyloidogenic regions. LICTOR, instead, aims at finding hotspots responsible for LC toxicity in AL amyloidosis, starting from the known clinical phenotype of LC sequences and following the assumption that SMs are the key determinants of LC proteotoxicity. Indeed, our approach uses an innovative encoding scheme to express LC sequences as the difference with respect to the germline. Then, this strategy is applied to extract sequence and structural features, and to investigate their role in the determination of LC proteotoxicity. Indeed, we showed that specific features (sequence AMP or structural MAP and DAP features) that provide the largest information gain to LICTOR harbour crucial information to accurately predict LC phenotype and can thus be regarded as effective AL molecular determinants. In fact, through the information gain feature selection process, we identified a set of features characterized by the strongest association with the LC phenotype, which, remarkably, were mainly located at the dimeric interface of the LC structure. This finding further emphasizes the 
key role of the structural context of SMs as drivers of LC proteotoxicity.

We also performed a comparison with AGGRESCAN, PASTA and WALTZ, using the aggregation propensities $\left(\mathrm{P}_{\mathrm{A}}\right.$ for AGGRESCAN, $\mathrm{P}_{\mathrm{P}}$ for PASTA and $\mathrm{P}_{\mathrm{W}}$ for WALTZ) provided by the respective programs to construct three classifiers. Interestingly, in all three cases tox sequences are significantly more aggregation prone than nox sequences (for AGGRESCAN mean $\mathrm{P}_{\mathrm{A}}=-8.53 \pm 4.2$ vs $-7.44 \pm 3.8, p$-value $<0.0001$ unpaired $t$-test; for PASTA mean $\mathrm{P}_{\mathrm{P}}=-6.35 \pm 1.08$ vs $-5.82 \pm 1.15, p$-value $=$ $<0.0001$ unpaired $t$-test; and for WALTZ mean $\mathrm{P}_{\mathrm{W}}=97.78 \pm$ 0.83 vs $97.49 \pm 0.89, p$-value $=0.009$ unpaired $t$-test). However, given the considerable overlap between the propensity distributions, classifiers based on aggregation propensity have a rather limited accuracy (AGGRESCAN accuracy $=0.59$, PASTA accuracy $=0.68$, WALTZ accuracy $=0.64)$. Nevertheless, these approaches indicate that toxic sequences are more prone to aggregate than non-toxic ones, a fact that is mirrored in LICTOR by the identification of SMs clustering at the dimer interface as divers of proteotoxicity in AL.

Previous studies have analysed the fibril formation propensity of LC variable domains (VL), showing that destabilizing mutations at specific structural sites correlate with increased amyloid fibril formation ${ }^{49-51}$. Additional reports have also compared the stability and fibril formation propensity of variable domains of toxic LC sequences associated with $\mathrm{AL}$ and non-toxic ones associated with $\mathrm{MM}^{52,53}$, suggesting that specific mutations could induce a destabilization in toxic LCs. However, as pointed out in a recent analysis of full-length LCs associated with $\mathrm{AL}$ or $\mathrm{MM}^{27}$, despite significant differences in some properties such as the melting temperature $\left(T_{\mathrm{m}}\right)$, it is not possible to unequivocally differentiate between pathogenic and non-pathogenic LCs based on a single biophysical property. Only flexibility and susceptibility to protease cleavage emerged as discriminative factors of proteotoxicity.

Other reports have suggested that germline proteins are more stable than the corresponding pathological LCs ${ }^{39,54}$. Analysing the variable domains of the pathogenic light chain AL09 and its corresponding germline $\mathrm{\kappa I} \mathrm{O} 18 / \mathrm{O} 8$, Baden et al., noticed that a non-conservative SM at the dimeric interface of AL09 (Y87H, according to Kabat numbering scheme), induced an altered dimer interface, characterized by a $90^{\circ}$ rotation with respect to the canonical homodimeric structure of the germline counterpart. Interestingly, the same position (position 99 according to our sequential numbering scheme, corresponding to position 87 in Kabat-Chothia numbering) was identified as one of the most important structural features (Fig. 2d and Supplementary Data 8) used by LICTOR to predict LC toxicity.

Cryo-EM structures of LC amyloid fibrils ${ }^{55,56}$ show an interesting rearrangement in the region comprising of the intrachain disulfide bond; namely, in folded LCs these two cysteines connect parallel $B$-strands, while in the amyloid fibrils the two B-strands are antiparallel. These conformational rearrangements break the intrachain interaction between CDR1 and CDR3, as well as, the intrachain interactions between FR2 and end of FR3. Furthermore, the dimerization interface of the folded LC is disrupted in the fibrils, as they are on the opposite side of the fibril layer. These findings are in line with our results that suggest that SM located at the LC homodimeric interface may impair the structural integrity of the protein-protein interface and/or induce local instability of the monomer, with consequent triggering of LC misfolding and the generation of toxic species.

The starting hypothesis that SMs are key determinants of AL, the accuracy of LICTOR and of our in silico findings were also experimentally confirmed in C. elegans, a validated in vivo model for assessing LC toxicity. We demonstrated that germline LCs are not able to induce proteotoxicity in vivo, validating the assumption that naive LC sequences acquire the toxic phenotype in AL during affinity maturation. Notably, as predicted by LICTOR, the toxic phenotype of an LC was abolished by reverting a single SM.

Taken together, these findings confirm the accuracy and robustness of our in silico approach in the identification of toxic and non-toxic LCs and suggest its usefulness as a diagnostic instrument for AL.

Machine learning relies on data. Larger datasets of LC sequences would, therefore, be beneficial for validation, as well as for the improvement of LICTOR accuracy. However, collecting large numbers of toxic LC sequences is difficult due to the low prevalence of the disease. We believe that the application of LICTOR as a possible diagnostic tool could encourage clinicians to obtain - and make available to the public- $\mathrm{LC}$ sequences of $\mathrm{AL}$ patients, thus increasing the size of LC sequence databases and consequently allowing improvement of LICTOR accuracy. Furthermore, other factors such as the increased protein dynamics of toxic $\mathrm{LCs}^{27}$ or the generation of LC glycosylation sites by SMs, may be included among the predictive features to improve LC toxicity classification, as suggested by previous reports ${ }^{51,57}$. This would not only improve the accuracy of LICTOR, but also deepen our understanding of AL determinants and shed light on the complex mechanism of AL development.

In conclusion, LICTOR represents the first method for the accurate prediction LC toxicity from their sequence, allowing the timely identification of high-risk patients, such as MGUS subjects likely to progress to AL. Using LICTOR can thus promote a closer monitoring for AL development and foster early treatment and better patients' prognosis. Finally, LICTOR may be used together with other recently proposed strategies, such as the differential recruitment efficacy of patient-derived full-length LCs by synthetic amyloid fibril ${ }^{58}$, to predict the risk of AL development. Our approach may, furthermore, guide the development of novel predictive tools useful for other diseases, such as cancer, in which the prognosis may depend on SMs of specific tumour-linked proteins. LICTOR is available as a webservice at http://lictor.irb. usi.ch.

\section{Methods}

Dataset. The database used in the training was composed of 428 tox and 590 nox sequences of the $\lambda$ isotype collected from AL-Base (http://albase.bumc.bu.edu). Furthermore, it contained 57 nox $\lambda$ LC sequences that we collected at the Institute for Research in Biomedicine (IRB-DB), known to be non-toxic in the context of AL. The 1075 sequences were automatically aligned using a progressive KabatChothia numbering scheme (http://www.bioinf.org.uk/abs/). According to this scheme, for example, the CDR1 of a given LC with Kabat-Chothia numbering 30A, $30 \mathrm{~B}, 30 \mathrm{C}, 30 \mathrm{D}, 30 \mathrm{E}$ and $30 \mathrm{~F}$ was assigned $31,32,33,34,35$ and 36. For the ALBase sequences, germline information was taken from the database, while for IRB-DB LCs, the germline was assessed with an in-house script. Next, germline (GL) sequences were reconstructed using the IMGT database ${ }^{23}$.

The germline sequences were aligned with the same numbering scheme used for the LCs. Next, each LC in the dataset was compared with the corresponding germline to identify all SMs, with the differences encoded using an X for unmutated positions and the LC amino acid for SMs; this sequence was referred to as $S_{\text {mut }}$. For example, an LC with sequence SYELTQPP and a corresponding germline with the sequence SYVLTQPP was encoded as XXEXXXXX since there is an SM $(V \rightarrow E)$ at position 3. To compare the presence of SMs in $S_{\text {mut }}$ at each position $i$ in the Kabat-Chothia numbering scheme, the following four quantities were computed:

- $\operatorname{tox}_{\mathrm{NM}}{ }_{\mathrm{NM}}$ the number of toxic sequences without an SM at position $i$

- $\operatorname{tox}_{\mathrm{M}}^{i}$ - the number of toxic sequences with an SM at position $i$;

- $\operatorname{nox}^{i} \mathrm{NM}$ - the number of non-toxic sequences without an SM at position $i$

- $\operatorname{nox}_{M}^{i}$ - the number of non-toxic sequences with an SM at position $i$.

Statistical analysis. The fisher.test function in $\mathrm{R}$ version 3.5.1 with the arguments conf.int $=$ TRUE and conf.level $=0.95$ was used to assess significant differences in SMs between toxic and non-toxic sequences. The OR between $\operatorname{tox}_{M}^{i} / \operatorname{tox}_{\mathrm{NM}}^{i}$ and 
$\operatorname{nox}_{M}^{i} /$ nox $^{i}$ M was computed as

$$
\mathrm{OR}_{\text {tox }-\mathrm{nox}}^{i}=\frac{\operatorname{tox}_{\mathrm{M}}^{i} / \operatorname{tox}_{\mathrm{NM}}^{i}}{\operatorname{nox}_{\mathrm{M}}^{i} / \operatorname{nox}_{\mathrm{NM}}^{i}}
$$

$\mathrm{OR}=1$ indicates that the event under study (i.e. the frequency of mutations at position $i$ ) is equally likely in the two groups (e.g. tox vs nox). OR $>1$ indicates that the event is more likely in the first group (tox). OR $<1$ indicates that the event is more likely in the second group (nox). The t.test function in $\mathrm{R}$ version 3.5.1 was used to evaluate whether the PDSM differed between the tox, nox and hdnox datasets. Data from C. elegans-based assays were analysed using GraphPad Prism 8.2.1 software by one-way ANOVA and Dunn's post-test analysis. A p-value $<0.05$ was considered significant.

Predictor variables used by the machine learners. Given a sequence, the following three features were extracted:

Amino acid at each mutated position (AMP). From a sequence $S_{\text {mut }}$, a list of predictor variables was extracted, each describing the type of amino acid added by the SM at a given position or the absence of a mutation at the position. Thus, each of these variables was a pair (position, amino acid), where we used the letter "X" instead of the amino acid at the positions for which no SMs were present.

Monomeric amino acid pairs (MAP). LCs share a conserved 3D structure. Therefore, pairs of interacting residues were defined as amino acids having a distance between the respective $C \beta$ atoms less than $7.5 \AA$ in the X-ray structure (PDB ID: 2OLD).

Dimeric amino acid pairs (DAP). Similarly, pairs of residues that interact at the LC-LC interface were defined using the 2OLD LC homodimeric X-ray structure. Two residues belonging to different chains were considered to interact if the distance between their $\mathrm{C} \beta$ atoms was less than $7.5 \AA$.

Machine learning algorithms. Weka 3.8.1 28 implementation was used for the four machine learning algorithms (Bayesian network, logistic regression, J48 and random forest) to solve the classification task. For all algorithms, the default Weka parameters were used. The algorithms were evaluated by performing 10 -fold crossvalidation over the dataset. The performance of each algorithm was: first, assessed using only one family of features (e.g. AMP, MAP and DAP, for a total of three combinations); second, the three families were combined into pairs (e.g. AMP U MAP, for a total of three combinations); third, all three families were combined together. This led to a total of 7 (feature configurations) $\times 4$ (algorithms) $=28$ prediction experiments. Moreover, each of the 28 experiments was performed with and without the balancing of the training set with $\mathrm{SMOTE}^{29}$ on the toxic sequences so that the number of toxic instances was equal to the number of non-toxic instances in the training set during each of the ten cross-validations used in the evaluation. This led to $28 \times 2$ (with/without SMOTE) $=56$ total experiments.

Prediction performance. The various prediction algorithms were assessed by computing the following classification errors: (i) Type-I misclassifications, indicating toxic sequences incorrectly classified as non-toxic (false negative, FN) and (ii) Type-II misclassifications, indicating non-toxic sequences misclassified as toxic (false positive, FP). The correct classifications were instead indicated by the number of true positives, TP (a toxic sequence correctly classified) and true negatives, TN (a non-toxic sequence correctly classified). Based on TP, TN, FP and $\mathrm{FN}$, the following metrics were used to evaluate the performance of our classifiers:

- Area under the receiver operating characteristic curve (AUC): The AUC is used to assess the performance of a two-class classifier (such as that in our study) and is equal to the probability that the classifier will rank a randomly chosen positive instance (in our case, a toxic sequence) higher than a randomly chosen negative instance (a non-toxic sequence). A random classifier has an $\mathrm{AUC}=0.5$, while the $\mathrm{AUC}$ is 1.0 for a perfect classifier.

- Sensitivity: Computed as TP/(TP $+\mathrm{FN})$, this represents the percentage of toxic sequences correctly identified by the classifier.

- Specificity: Computed as TN/(TN + FP), this represents the percentage of non-toxic sequences correctly identified by the classifier.

- Accuracy: Computed as $(\mathrm{TP}+\mathrm{TN}) /(\mathrm{TP}+\mathrm{FP}+\mathrm{TN}+\mathrm{FN})$, this represents the overall percentage of correctly classified sequences.

- Balanced accuracy: Computed as (specificity + sensitivity)/2, this represents the arithmetic mean of sensitivity and specificity.

- F1 score: Computed as $2 \mathrm{TP} /(2 \mathrm{TP}+\mathrm{FP}+\mathrm{FN})$, this represents the harmonic mean of the sensitivity and the precision, which is computed as the number of $\mathrm{TP} /(\mathrm{TP}+\mathrm{FN})$. $\mathrm{F} 1=1$ indicates perfect precision and sensitivity, while $\mathrm{F} 1=0$ represents the lowest possible value achieved if either the precision or the sensitivity is 0 .

Youden index. The Youden $(J)$ index was used to validate the effectiveness of the predictors and to find the optimal cut-off point to separate toxic LCs associated with the disease from non-toxic LCs using the following formula:

$$
J=\max _{c}[\operatorname{Se}(c)+\operatorname{Sp}(c)-1]
$$

Information gain feature selection. The InfoGainAttributeEval filter implemented in Weka 3.8.1 $1^{34}$ was used to remove all features that did not contribute to the information available for the prediction of the sequence type. All features having an information gain less than 0.01 were removed. Given the computational cost of this procedure, this experiment was performed for the best-performing algorithm and configuration identified in the previous 56 experiments. The full list of ranked features is shown in Supplementary Data 8.

Tox153 sequence. The LC sequence was sequenced from circularized cDNA obtained from bone marrow cells as previously described ${ }^{59}$. First, $1 \mu \mathrm{L}$ of circularized cDNA was mixed with $5 \mu \mathrm{L} 5 \mathrm{X}$ Q $5^{\oplus}$ Reaction Buffer (New England Biolabs), $0.5 \mu \mathrm{L} 5 \mathrm{X} \mathrm{Q} 5^{\oplus}$ High GC Enhancer (New England Biolabs), $0.5 \mu \mathrm{L}$ dNTPs mix $(25 \mathrm{mM}), 1.25 \mu \mathrm{L}$ primers $(10 \mu \mathrm{M}$ each $)$ and $0.25 \mu \mathrm{L}$ Q5 ${ }^{\circledast}$ High-Fidelity DNA Polymerase $(2 \mathrm{U} / \mu \mathrm{L})$ (New England Biolabs) in a final volume of $25 \mu \mathrm{L}$. Then the sample was denatured for $1 \mathrm{~min}$ at $98^{\circ} \mathrm{C}$ and 35 PCR cycles were performed under the following conditions: $98^{\circ} \mathrm{C}(10 \mathrm{~s}), 67^{\circ} \mathrm{C}(20 \mathrm{~s}), 72^{\circ} \mathrm{C}(40 \mathrm{~s})$ and final extension $72^{\circ} \mathrm{C}(2 \mathrm{~min})$. Lambda light chains were amplified with the specific primers $\lambda-\mathrm{C}_{\mathrm{LA}}$ (5'-AGT GTG GCC TTG TTG GCT TG-3) and $\lambda$ - $\mathrm{C}_{\mathrm{LB}}$ (5'-GTC ACG CAT GAA GGG AGC AC-3), and a library of unique sequences was obtained by a Zero Blunt ${ }^{\oplus O P O}{ }^{\oplus}$ PCR Cloning Kit (Life Technologies) and subsequent analysis of single colonies.

Acquisition, storage and use of biological samples were approved by the institutional review board (Comitato Etico Area di Pavia). Written informed consent was received from participants prior to inclusion in the study. The study was conducted in accordance with the Declaration of Helsinki.

Protein production and purification. Tox153, tox153V52L and tox153V52LA56G were custom expressed in mammalian cell lines (Expi293F), purified by affinity purification column and analysed by SDS-PAGE and western blot by GenScript (New Jersey, USA). H6GL, H9GL, Tox153GL and H18 were expressed in mammalian cell lines (Expi293F), purified by HiTrap ${ }^{\circledR}$ LambdaFabSelect (GE Healthcare) and analysed by SDS-PAGE.

Effect of LCs on C. elegans. Bristol N2 nematodes were obtained from the C. elegans Genetic Center (CGC, University of Minnesota, Minneapolis, MN) and propagated at $20^{\circ} \mathrm{C}$ on solid nematode growth medium (NGM) seeded with Escherichia coli OP50 (CGC) for food. Worms were incubated with $100 \mu \mathrm{g} / \mathrm{mL}$ tox 153 wild-type protein, tox153V52L or tox153V52LA56G (100 worms $/ 100 \mu \mathrm{L})$ in $10 \mathrm{mM}$ phosphate-buffered saline (PBS, pH 7.4) $)^{8,9}$. Hydrogen peroxide $(1 \mathrm{mM})$ was administered under dark conditions as a positive control and $10 \mathrm{mM}$ PBS (pH 7.4) as a negative control (vehicle). After $2 \mathrm{~h}$ of incubation with orbital shaking, worms were transferred onto NGM plates seeded with OP50 E. coli. The pharyngeal pumping rate, measured by counting the number of times the terminal bulb of the pharynx contracted over a $1 \mathrm{~min}$ interval, was scored $24 \mathrm{~h}$ later.

Reporting summary. Further information on research design is available in the Nature Research Reporting Summary linked to this article.

\section{Data availability}

Nox/tox sequences can be downloaded at https:/github.com/mauragarofalo/LICTOR/ data. Light chains can be downloaded from Amyloid Light-chain Database: http://albase. bumc.bu.edu. Source data are provided with this paper.

\section{Code availability}

LICTOR source code is available at: https://github.com/mauragarofalo/LICTOR/code. Weka 3.8.1 can be downloaded at https://waikato.github.io/weka-wiki/ downloading_weka/.

Received: 21 April 2020; Accepted: 23 May 2021; Published online: 10 June 2021

\section{References}

1. Merlini, G. \& Bellotti, V. Molecular mechanisms of amyloidosis. N. Engl. J. Med. 349, 583-596 (2003).

2. Lavatelli, F. et al. A novel approach for the purification and proteomic analysis of pathogenic immunoglobulin free light chains from serum. Biochim. Biophys. Acta 1814, 409-419 (2011).

3. Lousada, I., Comenzo, R. L., Landau, H., Guthrie, S. \& Merlini, G. Light chain amyloidosis: patient experience survey from the Amyloidosis Research Consortium. Adv. Ther. 32, 920-928 (2015). 
4. Palladini, G. \& Merlini, G. What is new in diagnosis and management of light chain amyloidosis? Blood 128, 159-168 (2016).

5. Merlini, G. et al. Systemic immunoglobulin light chain amyloidosis. Nat. Rev. Dis. Primers 4, 38 (2018).

6. Grogan, M., Dispenzieri, A. \& Gertz, M. A. Light-chain cardiac amyloidosis: strategies to promote early diagnosis and cardiac response. Heart 103, 1065-1072 (2017).

7. Gertz, M. A. Immunoglobulin light chain amyloidosis diagnosis and treatment algorithm 2018. Blood Cancer J. 8, 44 (2018).

8. Merlini, G. Determining the significance of MGUS. Blood 123, 305-307 (2014).

9. Blancas-Mejia, L. M. \& Ramirez-Alvarado, M. Systemic amyloidoses. Annu. Rev. Biochem. 82, 745-774 (2013).

10. Wang, P. et al. Real-time automatic detection system increases colonoscopic polyp and adenoma detection rates: a prospective randomised controlled study. Gut 68, 1813-1819 (2019).

11. Shen, L. et al. Deep learning to improve breast cancer detection on screening mammography. Sci. Rep. 9, 12495 (2019).

12. Yoo, S., Gujrathi, I., Haider, M. A. \& Khalvati, F. Prostate cancer detection using deep convolutional neural networks. Sci. Rep. 9, 19518 (2019).

13. Saltz, J. et al. Spatial organization and molecular correlation of tumorinfiltrating lymphocytes using deep learning on pathology images. Cell Rep. 23, 181-193 e187 (2018).

14. Corredor, G. et al. Spatial architecture and arrangement of tumor-infiltrating lymphocytes for predicting likelihood of recurrence in early-stage non-small cell lung cancer. Clin. Cancer Res. 25, 1526-1534 (2019).

15. Olivecrona, M., Blaschke, T., Engkvist, O. \& Chen, H. Molecular de-novo design through deep reinforcement learning. J. Cheminform. 9, 48 (2017).

16. Kadurin, A., Nikolenko, S., Khrabrov, K., Aliper, A. \& Zhavoronkov, A druGAN: an advanced generative adversarial autoencoder model for de novo generation of new molecules with desired molecular properties in silico. Mol. Pharm. 14, 3098-3104 (2017).

17. Costello, J. C. et al. A community effort to assess and improve drug sensitivity prediction algorithms. Nat. Biotechnol. 32, 1202-1212 (2014).

18. Ali, M., Khan, S. A., Wennerberg, K. \& Aittokallio, T. Global proteomics profiling improves drug sensitivity prediction: results from a multi-omics, pan-cancer modeling approach. Bioinformatics 34, 1353-1362 (2018).

19. Cortes-Ciriano, I. et al. Improved large-scale prediction of growth inhibition patterns using the NCI60 cancer cell line panel. Bioinformatics 32, 85-95 (2016).

20. Rajkomar, A., Dean, J. \& Kohane, I. Machine learning in medicine. N. Engl. J. Med. 380, 1347-1358 (2019).

21. Bodi, K. et al. AL-Base: a visual platform analysis tool for the study of amyloidogenic immunoglobulin light chain sequences. Amyloid 16, 1-8 (2009).

22. Dispenzieri, A., Gertz, M. A. \& Buadi, F. What do I need to know about immunoglobulin light chain (AL) amyloidosis? Blood Rev. 26, 137-154 (2012).

23. Brochet, X., Lefranc, M. P. \& Giudicelli, V. IMGT/V-QUEST: the highly customized and integrated system for IG and TR standardized V-J and V-D-J sequence analysis. Nucleic Acids Res. 36, W503-W508 (2008).

24. Sheskin, D. Handbook of Parametric and Nonparametric Statistical Procedures. 4th edn. (Chapman \& Hall/CRC, 2007).

25. DeKosky, B. J. et al. Large-scale sequence and structural comparisons of human naive and antigen-experienced antibody repertoires. Proc. Natl Acad. Sci. USA 113, E2636-E2645 (2016).

26. Makino, D. L., Henschen-Edman, A. H., Larson, S. B. \& McPherson, A. Bence Jones KWR protein structures determined by X-ray crystallography. Acta Crystallogr. D. Biol. Crystallogr. 63, 780-792 (2007).

27. Oberti, L. et al. Concurrent structural and biophysical traits link with immunoglobulin light chains amyloid propensity. Sci. Rep. 7, 16809 (2017).

28. Hall, M. F. E., Holmes, G., Pfahringer, B., Reutemann, P. \& Witten, I. H. The WEKA data mining software: an update. SIGKDD Explor. Newsl. 11, 10-18 (2009).

29. Chawla, N. V., Bowyer, K. W., Hall, L. O. \& Kegelmeyer, W. P. SMOTE: synthetic minority over-sampling technique. J. Artif. Intell. Res. 16, 321-357 (2002).

30. Abraham, R. S. et al. Immunoglobulin light chain variable (V) region genes influence clinical presentation and outcome in light chain-associated amyloidosis (AL). Blood 101, 3801-3808 (2003).

31. Comenzo, R. L., Zhang, Y., Martinez, C., Osman, K. \& Herrera, G. A. The tropism of organ involvement in primary systemic amyloidosis: contributions of Ig $\mathrm{V}(\mathrm{L})$ germ line gene use and clonal plasma cell burden. Blood 98, 714-720 (2001).

32. Kourelis, T. V. et al. Clarifying immunoglobulin gene usage in systemic and localized immunoglobulin light-chain amyloidosis by mass spectrometry. Blood 129, 299-306 (2017).

33. Youden, W. J. Index for rating diagnostic tests. Cancer 3, 32-35 (1950).

34. Witten, I. H., Frank, E. \& Hall, M. A. Data Mining: Practical Machine Learning Tools and Techniques. 3rd edn. (Morgan Kaufmann, 2011).
35. Diomede, L. et al. A Caenorhabditis elegans-based assay recognizes immunoglobulin light chains causing heart amyloidosis. Blood 123, 3543-3552 (2014)

36. Diomede, L. et al. Cardiac light chain amyloidosis: the role of metal ions in oxidative stress and mitochondrial damage. Antioxid. Redox Signal. 27, 567-582 (2017).

37. Perfetti, V. et al. The repertoire of lambda light chains causing predominant amyloid heart involvement and identification of a preferentially involved germline gene, IGLV1-44. Blood 119, 144-150 (2012).

38. Maritan, M. et al. Inherent biophysical properties modulate the toxicity of soluble amyloidogenic light chains.J. Mol. Biol. 432, 845-860 (2020).

39. Baden, E. M., Randles, E. G., Aboagye, A. K., Thompson, J. R. \& RamirezAlvarado, M. Structural insights into the role of mutations in amyloidogenesis. J. Biol. Chem. 283, 30950-30956 (2008).

40. del Pozo Yauner, L. et al. Influence of the germline sequence on the thermodynamic stability and fibrillogenicity of human lambda 6 light chains. Proteins 72, 684-692 (2008).

41. Davis, D. P. et al. Both the environment and somatic mutations govern the aggregation pathway of pathogenic immunoglobulin light chain. J. Mol. Biol. 313, 1021-1034 (2001).

42. de Groot, N. S., Castillo, V., Grana-Montes, R. \& Ventura, S. AGGRESCAN method, application, and perspectives for drug design. Methods Mol. Biol. 819, 199-220 (2012).

43. Walsh, I., Seno, F., Tosatto, S. C. \& Trovato, A. PASTA 2.0: an improved server for protein aggregation prediction. Nucleic Acids Res. 42, W301-W307 (2014).

44. Maurer-Stroh, S. et al. Exploring the sequence determinants of amyloid structure using position-specific scoring matrices. Nat. Methods 7, 237-242 (2010).

45. Tartaglia, G. G., Cavalli, A., Pellarin, R. \& Caflisch, A. Prediction of aggregation rate and aggregation-prone segments in polypeptide sequences. Protein Sci. 14, 2723-2734 (2005).

46. Tartaglia, G. G. \& Vendruscolo, M. The Zyggregator method for predicting protein aggregation propensities. Chem. Soc. Rev. 37, 1395-1401 (2008).

47. Fernandez-Escamilla, A. M., Rousseau, F., Schymkowitz, J. \& Serrano, L. Prediction of sequence-dependent and mutational effects on the aggregation of peptides and proteins. Nat. Biotechnol. 22, 1302-1306 (2004).

48. Thompson, M. J. et al. The 3D profile method for identifying fibril-forming segments of proteins. Proc. Natl Acad. Sci. USA 103, 4074-4078 (2006).

49. Hurle, M. R., Helms, L. R., Li, L., Chan, W. \& Wetzel, R. A role for destabilizing amino acid replacements in light-chain amyloidosis. Proc. Natl Acad. Sci. USA 91, 5446-5450 (1994).

50. Raffen, R. et al. Physicochemical consequences of amino acid variations that contribute to fibril formation by immunoglobulin light chains. Protein Sci. 8, 509-517 (1999).

51. Stevens, F. J. Four structural risk factors identify most fibril-forming kappa light chains. Amyloid 7, 200-211 (2000).

52. Wall, J. et al. Thermodynamic instability of human lambda 6 light chains: correlation with fibrillogenicity. Biochemistry 38, 14101-14108 (1999).

53. Kobayashi, Y. et al. Decreased amyloidogenicity caused by mutational modulation of surface properties of the immunoglobulin light chain BRE variable domain. Biochemistry 53, 5162-5173 (2014).

54. Baden, E. M. et al. Altered dimer interface decreases stability in an amyloidogenic protein. J. Biol. Chem. 283, 15853-15860 (2008).

55. Swuec, P. et al. Cryo-EM structure of cardiac amyloid fibrils from an immunoglobulin light chain AL amyloidosis patient. Nat. Commun. 10, 1269 (2019).

56. Radamaker, L. et al. Cryo-EM structure of a light chain-derived amyloid fibril from a patient with systemic AL amyloidosis. Nat. Commun. 10, 1103 (2019).

57. Kumar, S. et al. Assay to rapidly screen for immunoglobulin light chain glycosylation: a potential path to earlier AL diagnosis for a subset of patients. Leukemia 33, 254-257 (2019).

58. Martin, E. B. et al. Differential recruitment efficacy of patient-derived amyloidogenic and myeloma light chain proteins by synthetic fibrils-A metric for predicting amyloid propensity. PLoS ONE 12, e0174152 (2017).

59. Perfetti, V. et al. Inverse polymerase chain reaction for cloning complete human immunoglobulin variable regions and leaders conserving the original sequence. Anal. Biochem. 239, 107-109 (1996).

\section{Acknowledgements}

This study was supported by grants from the Swiss National Science Foundation (31003A-166472 and 31003A 182270) to A.C. and L.V. and from the Italian Ministry of Health (RF-2013-02355259 and RF-2016-02361756) to L.D. C. elegans and OP50 E. coli were provided by the CGC, which is funded by NIH Office Research Infrastructure Programs (P40 OD010440). We would like to acknowledge the use of the Boston University ALBase, supported by HL68705, and Dr. Giampaolo Merlini and Dr. Mario Nuvolone, who provided bone marrow samples. 


\section{Author contributions}

A.C. and M.G. designed the research. M.G. performed data acquisition and analysis and drafted the manuscript. M.F., L.P., S.R., M.R. M.M.B, R.D.G., L.V., L.D. and A.L. provided data. M.G., L.P., S.R., M.F., M.M., J.S., R.D.G., M.P., O.M., A.L., L.D. and A.C. edited and approved the manuscript.

\section{Competing interests}

The authors declare no competing interests.

\section{Additional information}

Supplementary information The online version contains supplementary material available at https://doi.org/10.1038/s41467-021-23880-9.

Correspondence and requests for materials should be addressed to A.C.

Peer review information Nature Communications thanks Vittorio Bellotti and the other, anonymous, reviewer(s) for their contribution to the peer review of this work.
Publisher's note Springer Nature remains neutral with regard to jurisdictional claims in published maps and institutional affiliations.

(c) (i) Open Access This article is licensed under a Creative Commons Attribution 4.0 International License, which permits use, sharing, adaptation, distribution and reproduction in any medium or format, as long as you give appropriate credit to the original author(s) and the source, provide a link to the Creative Commons license, and indicate if changes were made. The images or other third party material in this article are included in the article's Creative Commons license, unless indicated otherwise in a credit line to the material. If material is not included in the article's Creative Commons license and your intended use is not permitted by statutory regulation or exceeds the permitted use, you will need to obtain permission directly from the copyright holder. To view a copy of this license, visit http://creativecommons.org/ licenses/by/4.0/.

(c) The Author(s) 2021 